\title{
Médiation(s) : quelques remarques sur un terme pluriel
}

Danielle Chini

\section{CpenEdition}

\section{Journals}

Édition électronique

URL : http://journals.openedition.org/asp/1901

DOI : 10.4000/asp. 1901

ISBN : 978-2-8218-0384-8

ISSN : 2108-6354

Éditeur

Groupe d'étude et de recherche en anglais de spécialité

Édition imprimée

Date de publication : 1 octobre 2001

Pagination : 133-142

ISSN : 1246-8185

\section{Référence électronique}

Danielle Chini, « Médiation(s) : quelques remarques sur un terme pluriel », ASp [En ligne], 31-33 | 2001, mis en ligne le 12 novembre 2010, consulté le 20 avril 2019. URL : http://journals.openedition.org/ asp/1901; DOI : 10.4000/asp.1901

Ce document a été généré automatiquement le 20 avril 2019.

Tous droits réservés 


\title{
Médiation(s) : quelques remarques sur un terme pluriel
}

\author{
Danielle Chini
}

1 Nul aujourd'hui ne niera que le concept de «médiation » est au cœur des préoccupations de la didactique des langues, à la médiation inhérente à toute relation pédagogique s'ajoutant le caractère particulier de l'objet d'apprentissage. Mais si la compréhension de ce que recouvre ce concept est essentielle pour tout enseignant de langue, la tâche se révèle encore plus compliquée dans le cas des langues de spécialité, où la langue étrangère n'est plus simplement objet d'apprentissage mais devient aussi vecteur d'un contenu autre, outil d'un développement technique ou professionnel, et acquiert ainsi un statut réellement fonctionnel. Ne pourrait-on pas dire alors qu'elle joue un rôle de médiation entre l'apprenant et son domaine de spécialité ? Mais faut-il dans ce cas parler de médiation ou de médiatisation ou encore de simple outil de communication et d'interaction? Tous ces concepts voisins se confondent souvent et le constat qui s'impose est celui de la grande difficulté qu'il y a à cerner le concept de «médiation », pourtant très largement utilisé aujourd'hui dans le domaine qui est le nôtre, et la nécessité qui en découle de chercher à le mieux définir.

2 Cette question, cependant, dépasse largement le cadre de l'enseignement des langues de spécialité, dans la mesure où le terme de "médiation» est utilisé dans de nombreux autres domaines, de la sphère scientifique comme de la vie courante, et qu'on y est confronté au même problème de délimitation. Si dans tous les cas il sert bien à désigner un intermédiaire, son extension est une preuve suffisante de la généralité du concept qu'il désigne et en conséquence de la difficulté qu'il y a à définir clairement les caractéristiques de ce qu'il recouvre.

3 La nécessité de clarification du terme ne concerne donc pas le seul enseignement d'anglais de spécialité. Dans le domaine plus large de la didactique des langues qui nous concernera ici, cette impression de flou est en fait générale et n'est pas sans poser problème en formation initiale des enseignants quand il s'agit de cerner ce qu'on entend par le principe désormais banal selon lequel «dans une méthodologie centrée sur l'apprenant, l'enseignant ne doit plus être un transmetteur de savoir mais un 
médiateur.» Face à cette affirmation, un étudiant préparant le Capes a un jour conclu : "le rôle de l'enseignant est donc devenu secondaire. " Cette réflexion est bien sûr la conséquence d'une interprétation sinon erronée, du moins très étroite du terme, qui ne prend en compte que le caractère apparemment subsidiaire du rôle de l'intermédiaire par rapport à la préexistence des deux pôles fondamentaux qu'il aide à relier, ici l'apprenant et la matière. Il n'en reste pas moins que la multiplicité des emplois du terme "médiation" d'une part, et des termes connexes d'autre part, porte à confusion. Comment distinguer, s'il faut les distinguer, les concepts de médiation, de guidage, de tutelle ou encore d'étayage? Comment délimiter les domaines respectifs de la médiation, des médias, de la médiatisation, ou encore de la remédiation? Autant de termes et de concepts qui mettent le futur enseignant, et parfois bien des collègues plus chevronnés, face à une pluralité dans laquelle ils ont beaucoup de mal à se retrouver, et qui ne leur permet pas de percevoir la cohérence du ou des concepts en jeu et, au-delà, du système dans son ensemble.

Plus grave encore, un traitement globalisant du concept de médiation, doublé d'une confusion entre médiation et médiatisation, conduit parfois à mettre sur le même plan des « ressources » les simples outils de médiation et les acteurs que sont, entre autres, les enseignants. Parle-t-on réellement de la même chose par exemple lorsqu'on parle de médiation par l'image et de médiation pédagogique? Dans une séquence multimédia, l'ordinateur joue-t-il vraiment le même rôle que l'enseignant en présentiel ? Cette confusion est fréquente dans les publicités pour didacticiels commerciaux, ou encore dans les discours journalistiques de vulgarisation, mais aussi trop souvent dans les discours officiels, en particulier dans ceux qui concernent les langues de spécialité, où l'enseignant devient "personne ressource" et où les nouvelles technologies sont présentées comme la solution miracle à tous nos problèmes. L'impression qui ressort de cette confusion, impression souvent répercutée par le grand public, est celle d'une instrumentalisation de l'enseignement et, par ricochet, de l'enseignant, dont l'expertise est dangereusement minimisée, tout particulièrement dans le cas de l'enseignement de spécialité, où la langue étrangère est au service des préoccupations professionnelles prioritaires du public concerné : tous les outils lui étant fournis, la tâche de l'enseignant serait largement facilitée, quand la présence de l'outil ne la rend pas totalement caduque.

On est là face au problème exposé dans le texte de présentation d'un colloque sur la médiation, organisé par le groupe UMR CNRS 6065, et qui s'est tenu à Rouen en décembre 2000, celui du brouillage des frontières:

De façon globale, est médiateur tout élément (vivant ou non) qui, s'intercalant entre deux autres, en modifie les relations. Cependant, si l'on s'en tient à une définition aussi générale, tout peut être, d'un point de vue ou d'un autre, médiateur de quelque chose. En particulier, dans le domaine des sciences humaines ou sociales, ce terme convient à toutes les situations étudiées, puisque la notion de médiation est chevillée au caractère social du monde et aux besoins de communication et d'action. Pour que deux entités humaines puissent participer à des échanges il leur faut un lien, un intermédiaire, un moyen terme. Quelle que soit la nature de celui-ci (objets, personnes, signes), on pourra toujours parler de médiation.

6 Toute intervention magistrale serait donc médiation. Bien sûr, on ne peut empêcher les mots d'avoir une vie propre qui échappe aux spécialistes en entrant dans le domaine public. La spécificité des termes techniques se dilue dans le sens commun. C'est bien pour cela qu'il convient de faire, tout particulièrement en formation d'enseignants, un effort 
de définition et de recherche de cohérence, car, comme le rappelle le texte mentionné cidessus, « un soin particulier doit être apporté à l'utilisation et j'ajouterai à la définition d'une notion qui risque de devenir peu heuristique parce que trop générale ».

\section{Plusieurs formes de médiation?}

7 Dans cette perspective, la question qu'il me semble fondamental d'aborder en didactique des langues est la suivante: doit-on considérer, comme le suggère la présence entre parenthèses d'un $S$ pluriel dans le titre de ce colloque, qu'il existe plusieurs formes de médiation et qu'on a donc affaire à un concept pluriel et à un terme polysémique? Doit-on considérer plus spécifiquement que les médiations pédagogique, langagière, technologique, pour ne mentionner que celles-là, constituent des phénomènes distincts, des dynamiques autonomes? C'est à ces questions que nous nous proposons d'apporter des éléments de réponse.

8 On ne peut tenter de définir le concept de médiation sans se placer dans un cadre constructiviste et tout particulièrement socio-constructiviste, et donc sans se référer aux travaux de Vygotski et Bruner. Pour eux, le développement cognitif de l'enfant, et de l'être humain en général, est fondamentalement dépendant, d'une part, des interactions sociales dans lesquelles il est engagé et, d'autre part, de la disponibilité d'outils sémiotiques permettant ces interactions, et donc tout particulièrement du langage. En effet :

Toutes les fonctions psychiques supérieures sont unies par une caractéristique commune, celle d'être des processus médiatisés, c'est-à-dire d'inclure dans leur structure, en tant que partie centrale et essentielle du processus dans son ensemble, l'emploi du signe comme un moyen fondamental d'orientation et de maîtrise des processus psychiques. (Vygotski $1985: 150)$

En d'autres termes, les processus cognitifs ne peuvent se développer, et par là même les connaissances se construire, que par le biais d'une médiation sociale. Si Bruner s'est essentiellement préoccupé de la construction du jeune enfant dans sa vie quotidienne et du rôle central d'étayage qu'y joue son entourage adulte, tout spécialement sa mère, Vygotski a fait porter sa réflexion sur l'importance du cadre éducatif, ce qui lui confère, pour nous, tout son intérêt. Grâce en particulier aux concepts d'interaction, de langage intérieur, de zone de proche développement, il a donné une véritable épaisseur ainsi qu'une assise théorique au concept de médiation. C'est parce que l'enseignant, par son interaction raisonnée avec l'élève, l'amène à questionner l'objet d'apprentissage, à rationaliser et verbaliser ce questionnement, et à mettre ainsi à jour ses représentations internes, qu'il le fait progresser et lui permet de se dépasser, de concrétiser sa Zone de Proche Développement, cet « espace potentiel de progrès » qu'il n'aurait pas pu atteindre seul.

\section{Conceptualisation}

Tout le problème pour la formation initiale des enseignants, mais aussi pour la formation continue, c'est que, le plus souvent, ces concepts novateurs ne sont introduits qu'au travers de leurs implications méthodologiques, pédagogiques et technologiques, sans que la logique théorique qui les sous-tend et les justifie ne soit clairement explicitée. Or, on le sait, il ne suffit pas de changer les termes pour faire évoluer les pratiques, ni d'introduire 
de nouvelles techniques pédagogiques pour créer une nouvelle dynamique. Les évolutions réelles ne sont possibles que si l'on parvient à mettre à jour et à transformer les représentations de la relation enseignement-apprentissage, pour la plupart inconscientes, que chacun d'entre nous, professeurs et élèves, s'est construites, en grande partie au travers de ses expériences personnelles. Sans cette mise à jour, sans cette réflexion initiale, les concepts nous échappent et prennent pour les uns et les autres des significations souvent bien différentes de leur valeur théorique initiale. Ce danger de dérive est particulièrement réel dans le cas de l'apprentissage scolaire des langues étrangères où les notions d'interaction et de langage, qui sont le moteur de la médiation cognitive, peuvent facilement se dissoudre dans l'autre concept flou par excellence de la didactique des langues, le concept de communication, qui donne à ses notions une dimension bien différente de celle que leur attribuait Vygotski.

11 Prenons l'exemple de l'interaction. Au sens cognitif, elle suppose bien plus que la participation observable des élèves à des jeux communicatifs dans la classe. Elle vise beaucoup plus que le développement de compétences de communication. Cela conduit à poser la question du rôle de la communication: objectif à atteindre, spécifique aux apprentissages linguistiques, mais aussi cadre nécessaire à la construction de stratégies cognitives, fonction qui dépasse le seul cadre des langues vivantes. Comme le rappelle Gaonac'h,

il se peut que la communication constitue en fait la situation optimale d'acquisition, en ce sens qu'elle aurait une fonction effective et incontournable dans le développement du processus d'acquisition de la langue. $(1987: 179)$

Dans la perspective de la médiation cognitive, la communication doit donc être abordée comme un moyen plus que comme une fin, ce qui ne peut qu'avoir des implications importantes sur le choix des situations mises en place dans la classe et des activités proposées.

13 On retrouve la même dualité fin / moyen en ce qui concerne le langage. Il est clair qu'il ne suffit pas de faire parler l'élève pour que le langage joue son rôle de médiation. Cela est vrai pour toutes les matières mais est d'autant plus prégnant pour un apprentissage qui a pour objectif la maitrise d'un nouveau système linguistique. Outre sa fonction d'outil de communication, largement mobilisée dans la classe de langue, ce qui fait du langage une des composantes fondamentales de la médiation, c'est, comme le rappelle Bronckart dans l'abstract de l'intervention qu'il a faite au colloque de Rouen,

... une propriété d'autoréflexivité illimitée qui rend possible la prise de conscience et l'analyse de ces dépendances [liées à l'activité langagière], qui permet l'abstraction et la généralisation, ou encore la décontextualisation, et partant de là, la prise de distance à l'égard de toute forme de déterminisme historico-sociosémiotique. (UMR : 2000)

14 C'est cet aspect du langage qui fonde le rôle médiatif du langage. C'est donc cet aspect que l'enseignant doit solliciter, tout particulièrement par le biais d'activités métalinguistiques et métacognitives dans une perspective de conceptualisation. Car, comme le précise Doly,

la conscience qu'implique [la métacognition] est moins celle, psychologique, qui clôt le sujet sur lui-même, que celle, épistémique, qui médiatise son rapport aux savoirs. (1997: 94)

15 La gestion de la médiation en classe de langue pose donc le problème de la relation entre la langue étrangère dans laquelle il faut développer une compétence communicative la plus autonome possible, à visée souvent instrumentale, et la langue maternelle, qui 
constitue certes notre moyen privilégié d'échange, mais qui est surtout intimement liée à nos représentations mentales, à la structuration de notre pensée et, bien sûr, à notre langage intérieur, support de la conceptualisation.

\section{Interaction langagière et médiation cognitive} 1. Tout d'abord, la médiation est une dynamique qui suppose l'adhésion des deux parties, ce qui signifie, d'une part, conscience parfaite chez l'enseignant des implications cognitives de son rôle de guidage, et, d'autre part, motivation et implication volontaire de l'apprenant, qui doit se voir comme sujet actif et accepter de ne pas tout attendre de l'enseignant. Les remarques de Doly sur les enfants en échec sont à cet égard tout à fait significatives :

Ces sujets manifestent une absence de concept de soi: ils ne se connaissent pas, ne savent pas ce qu'ils savent, or «il ne suffit pas d'avoir des compétences et stratégies, encore faut-il savoir qu'on les a pour pouvoir les utiliser à bon escient » (Wong). En effet, ils se caractérisent par une attribution externe des causes de leurs échecs (et réussites). D'une façon générale, ils ne mettent pas en rapport l'activité $\mathrm{du}$ sujet et le résultat obtenu. Ils ne se perçoivent pas comme responsables actuels et potentiels de leur conduite ni de leurs résultats. Ils n'acquièrent aucune conscience de leur compétence à gérer une tâche, ni de sentiment de leur autoefficacité : ils ne se connaissent pas comme sujet apprenant et ne se font pas confiance pour progresser en même temps qu'ils ne peuvent ni verbaliser leur activité, ni la conceptualiser pour en faciliter la réutilisation. $(1997: 37-38)^{1}$

2. De cette constatation découle la deuxième condition nécessaire à la réussite de la médiation. Le médiateur doit avoir pour objectif non seulement la mise en relation de l'apprenant et de la matière, mais la découverte et l'évaluation, par l'apprenant luimême, de ses propres démarches et aptitudes cognitives, "en référence ", comme le rappelle Prouchet, « aux deux acceptions du terme cognitif : rapport à la connaissance et développement des outils de pensée » (1999: 34). Pour reprendre les termes de Buzan, il faut passer de la " pédagogie de l'éponge » à la « pédagogie de la découverte », en d'autres termes,

au lieu d'enseigner à l'individu des faits concernant d'autres disciplines, [et de lui demander] d'en absorber, d'en apprendre et d'en retenir un maximum, il faut d'abord lui enseigner les faits qui le concernent (ses propres mécanismes d'acquisition, de pensée, de mémorisation, d'invention, solution de problèmes, etc.). (1984: 136-137)

La véritable médiation est donc double et doit se concevoir comme une maïeutique : elle doit mettre en relation l'apprenant et la matière mais, de manière encore plus importante, elle doit révéler l'apprenant à lui-même.

3. Cela impose une troisième condition : l'enseignant, bien que spécialiste de la matière, doit s'efforcer de faire émerger les savoirs et éviter d'imposer aux élèves une vérité préétablie. Cela signifie partir des élèves, de leurs représentations initiales, comme l'ont clairement illustré de nombreux travaux sur la construction des savoirs scientifiques (cf. par exemple Giordan \& de Vecchi 1994, Charpak et l'opération «La main à la pâte »). 
Dans le cas des langues vivantes, cela doit conduire à s'inscrire dans une logique métacognitive de language awareness.

21 4. Enfin, cette dynamique ne peut être mise en place sans un important travail de réflexion et de didactisation en amont de la classe, étape essentielle dans laquelle il faut distinguer, comme l'indique Walski,

entre une didactisation qui amène l'apprenant vers la découverte de stratégies et procédures et une analyse qui dicte ce qu'il doit faire ou trouver. (1999: 30)

Car, comme elle le précise par ailleurs,

... les seules réponses utiles ne sont-elles pas celles qui viennent en écho à de vraies questions? Tant que la tâche n'a pas amené l'apprenant à ce vrai questionnement intérieur, la condition n'est pas créée d'un apprentissage durable. (1993: 62)

Le travail de médiation ne se limite donc pas à l'intervention de l'enseignant dans la classe mais se situe tout autant en amont, lors du choix des outils et des vecteurs de la médiation que sont les supports et les média qu'il va utiliser, ou encore les activités qu'il va proposer aux apprenants, créant ainsi dans la classe une situation réellement interactive. Comme l'écrit Doly,

On n'agit pas sur les êtres. [...] Mais on peut agir sur les choses, créer des dispositifs, mettre en place des espaces, prévoir des moments où les êtres puissent se mettre en jeu eux-mêmes. [...] On ouvre des possibles, on fait circuler des objets et des questions,[...] on crée une relation de confiance par un regard positif et on fournit des outils dont l'autre peut s'emparer. (1997: 7)

De même, la médiation est inséparable d'une évaluation conduisant à une remédiation et permettant ainsi le suivi et le contrôle du cheminement cognitif des apprenants. Comme le montrent avec clarté les travaux de Viselthier sur les liens entre « conceptualisation et processus de remédiation dans l'apprentissage de l'allemand (1996), la situation de remédiation est une occasion particulièrement propice au questionnement par l'élève de ses propres processus mentaux, si l'on prend la peine de mettre en place une réflexion guidée, consciente et verbalisée de l'apprenant sur la genèse de ses erreurs. Il s'agit de « l'amener à découvrir, grâce aux circuits intérieurs qu'il emprunte pour parvenir à la résolution d'une tâche, sa richesse potentielle» (Viselthier 1998 : 28). Autrement dit, pour Viselthier, la médiation doit être vue comme « un moteur favorisant l'atteinte de la zone de proche développement. » Il s'agit ainsi de faire prendre conscience à l'apprenant des liens entre ses productions et ses activités mentales, en d'autres termes entre les buts à atteindre et les moyens dont il dispose pour les atteindre. Dans cette perspective, la remédiation n'est plus simplement le remède à l'erreur administré par l'enseignant-évaluateur, mais une re-médiation, un nouvel acte de médiation à part entière, le plus important peut-être, vu qu'il part obligatoirement de la production des apprenants et les conduit donc à un vrai questionnement de leurs représentations, initiant ainsi une accommodation des schèmes qu'ils se sont préalablement construits. Cette étape de re-médiation s'inscrit en outre, comme le montre clairement le schéma (cf. figure 1) proposé par Viselthier (1998), dans une dynamique située où s'articulent de manière raisonnée l'élaboration anticipatrice de l'avant-cours, les activités de guidage propres à l'intervention pédagogique, et la réflexion accommodatrice de l'après-cours. 
Figure 1. La médiation comme moteur favorisant l'atteinte de la « zone de proche développement » (Viselthier 1998)

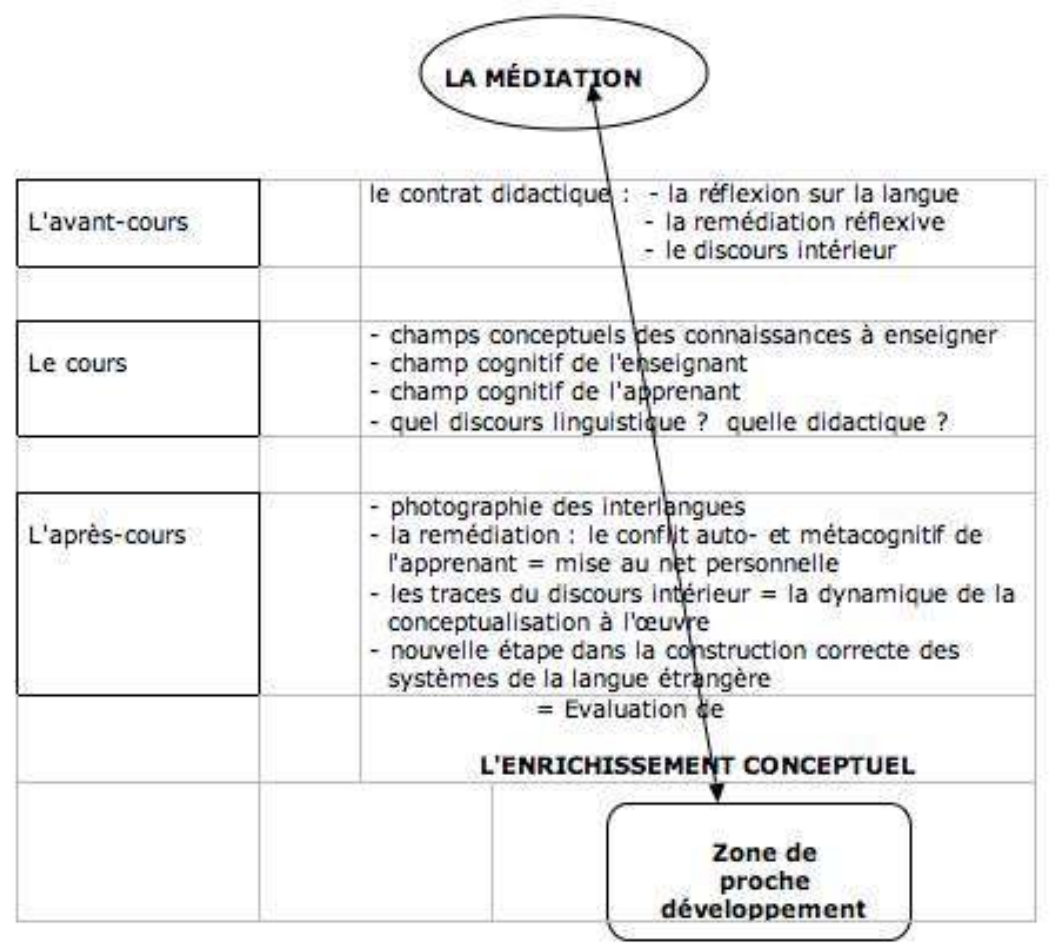

Illustration de la théorie de Vygotski créer les conditions de l'acquisition par l'apprenant des compétences de contrôle
nécessaires à la régulation autonome de ses activités langagières et de son propre apprentissage, autrement dit de créer les conditions de son autonomisation. En effet l'objectif fondamental de la médiation n'est pas, comme on l'entend souvent, sa disparition mais son intériorisation par l'apprenant sous la forme d'une capacité de contrôle et d'autorégulation, fondée sur son langage intérieur. Comme le rappelle Schneuwly, dans la logique vygotskienne, "c'est l'intégration d'outils sémiotiques socialement élaborés qui transforme le fonctionnement psychique et se trouve à la base de la construction de nouvelles fonctions psychiques" (1999:44). Entre médiation enseignante et autorégulation, il n'y a donc pas de différence de nature mais un continuum, dans lequel la re-médiation n'est qu'une étape. Cette intériorisation de la médiation s'accompagne bien sûr du passage progressif d'une gestion explicite à une mise en œuvre automatisée et donc implicite des processus de contrôle. C'est en fonction du rapport entre les degrés de médiation externe et de médiation interne que nécessitent ses activités et ses productions que s'évaluera l'autonomie de l'apprenant, ainsi qu'en fonction de la proportion de contrôle explicite, éventuellement verbalisé, et de mise en œuvre automatisée. 


\section{Conclusion}

Appréhendée dans cette perspective cognitive forte, la médiation, loin d'être plurielle, apparait donc comme une dynamique unique, même si elle est complexe, qui a partie liée avec la mise en place d'une approche métacognitive et conceptualisante et qui oblige à redéfinir la notion d'activité de l'apprenant. Dans cette perspective, les supports utilisés ainsi que les médias sollicités trouvent leur place comme des outils choisis par l'enseignant-médiateur pour construire la situation idéale qui permettra la mise en place conjointe des conditions d'une communication pragmatique signifiante et d'une interaction cognitive efficace. S'ils médiatisent la médiation, ils n'en sont pas l'origine et ne peuvent à eux seuls en assurer la réussite. La mise en place d'une dynamique de médiation dépend avant tout de celui qui pense et gère la situation d'apprentissage, que ce soit en continu comme l'enseignant dans le cas classique, ou en anticipation comme le concepteur de logiciels, et la réussite de cette dynamique suppose, on le voit, de la part de ce maître d'œuvre, une expertise accrue, fondée sur une véritable connaissance théorique des enjeux cognitifs et psycholinguistiques de l'activité d'apprentissage. Ce n'est qu'à ces conditions qu'on pourra mettre en place dans la classe une démarche médiatrice intégrée qui allie efficacité communicative et pertinence cognitive dans la perspective d'une véritable centration sur l'apprentissage, condition d'une réelle autonomisation de l'apprenant.

\section{BIBLIOGRAPHIE}

Bruner, Jérôme. 1983. Le développement de l'enfant. Savoir faire et savoir dire. Paris : Presses Universitaires de France.

Buzan, Tony. 1984. Une tête bien faite, 2e éd. Paris : Les Éditions d'Organisation.

Charpak, Georges. 1998. Enfants, chercheurs, citoyens. Paris : Editions Odile Jacob.

Doly, Anne-Marie. 1997. Métacognition et médiation. Clermont-Ferrand : CRDP Auvergne.

Gaonac'h, Daniel. 1987. Théories d'apprentissage et acquisition d'une langue étrangère. Paris : HatierCrédif.

Giordan, André et Gérard de Vecchi. 1994. Les origines du savoir : des conceptions des apprenants aux concepts scientifique. Lausanne : Delachaux et Niestlé.

Prouchet, Marc et Jean-Loup Héraud (dir.). 1999. Penser pour apprendre : regard critique sur l'éducation cognitive à l'école. Paris : Hatier.

Schneuwly, Bernard et Jean-François de Pietro. 1999. « Maîtriser un genre textuel en contexte scolaire : concepts, notions et démarches ». Actes des journées d'étude du GEPED, 12-13 mars 1999, Université Paris-Denis Diderot, 41-60.

UMR CNRS 6065. 2000. « Dynamiques Sociolangagières ». La Médiation : marquages en langue et en discours, Textes de présentation du colloque, 6-7-8 décembre 2000, Université de Rouen. 
Viselthier, Bernard. 1996. Dynamique de la conceptualisation et processus de remédiation dans l'apprentissage de l'allemand. Thèse de doctorat, Université Paris 3, Villeneuve d'Ascq : Presses Universitaires du Septentrion.

Viselthier, Bernard. 1998. «La médiation pédagogique comme révélateur du langage intérieur de l'apprenant et comme moteur favorisant l'atteinte de la zone de proche développement ». Actes des journées d'étude du GEPED, 20-21 mars 1998, Université Paris 7-Denis Diderot, 27-35.

Vygotski, Lev 1985 [1934] (traduction de F. Sève). Pensée et langage. Paris : Éditions Sociales.

Walski, Jennifer. 1993. « Du guidage en autonomie guidée ». Langues Modernes 93/1, 57-66.

Walski, Jennifer. 1999. « Le guidage : une démarche didactique pour aider l'apprenant dans le développement, la conceptualisation et la construction de la langue ». Actes des journées d'étude du GEPED, 12-13 mars 1999, Université Paris 7-Denis Diderot, 27-38.

\section{NOTES}

1. La référence à laquelle Doly fait allusion dans cette citation porte sur une contribution de B.Y.L. Wong «Metacognition and Learning Disabilities», In D.L. Forrest-Presley et al. (dir.), Metacognition, Cognition and Human Performance, vol. 2, New York : Academic Press, 1985.

\section{RÉSUMÉS}

La multiplicité des emplois directs ou dérivés du terme médiation est un signe du flou qui entoure ce concept, sa prise en compte se réduisant souvent à l'analyse de ses implications méthodologiques et technologiques. Or la médiation est un concept cognitif qui ne prend tout son sens que dans le cadre socio-constructiviste. Elle suppose avant tout un étayage social ainsi qu'un processus de contrôle et de re-médiation par le biais du langage, et vise la valorisation des capacités de l'apprenant et, à terme, son autonomisation. Dans le cadre de l'approche communicative cependant, on l'assimile trop souvent aux concepts d'interaction et de communication, confondant ainsi l'objectif instrumental de l'apprentissage et le cadre cognitif de son développement. Or toute interaction n'est pas cognitive. Pour qu'il en soit ainsi il faut créer, grâce à un guidage raisonné, une dynamique maïeutique susceptible de conduire l'apprenant à découvrir l'objet d'apprentissage mais aussi à comprendre et gérer ses propres processus mentaux. Entre médiation enseignante et autorégulation, il n'y a donc pas de différence de nature mais un continuum : la médiation constitue un processus complexe mais unique qu'il ne faut pas limiter aux divers supports et techniques qui lui servent d'outils.

"Mediation" is such a widely used concept in education, related to so many cognates, that it seems diverse and difficult to delimit. Yet it is a well-defined socio-constructivist concept that refers to a scaffolding process, aiming, through social interaction - mainly linguistic - and constant remediation, at developing the learner's capacities and autonomy. Its analysis certainly cannot be limited to assessing its methodological and technological implications. Within the communicative approach however, it is often confused with simple interactive communication, which blurs the fundamental difference between the functional aim of language learning and the 
cognitive framework that makes it possible. For interaction to be cognitively efficient, teacher guidance must be planned so that it makes the learners active in discovering both the foreign language and the working of their own mental skills: it should gradually lead them to plan and monitor their own performance. Ultimately, the difference between mediated teaching and self-regulation is only a matter of internalisation. Mediation then must be seen as a unique, if complex, process that should not be confused with or limited to the various media and activities required to implement it.

\section{INDEX}

Keywords : conceptualization, interaction, mediation (technological), remedial process, socioconstructivism

Mots-clés : conceptualisation, interaction, médiatisation, remédiation, socio-constructivisme

\section{AUTEUR}

\section{DANIELLE CHINI}

Danielle Chini, titulaire depuis 1996 d'une thèse intitulée « La problématique linguistique de l'enseignement de l'anglais dans le secondaire », est maître de conférences à l'université de Pau et des Pays de l'Adour, où elle assure des enseignements de linguistique et de didactique de l'anglais ainsi que de psycholinguistique. Elle dirige depuis le printemps 2000 le Groupe d'étude en psycholinguistique et didactique (GEPED), créé par le Professeur Danielle Bailly et initialement rattaché à l'Université Paris 7-Denis Diderot. Ses domaines de recherche privilégiés sont les relations entre la didactique de l'anglais d'une part et la linguistique et la psycholinguistique d'autre part, ainsi que toutes les questions qui se rattachent à la problématique de la conceptualisation dans l'apprentissage des langues. danielle.chini@univ-pau.fr 